\title{
Hidden Details of Negotiation: The Mechanics of Reality-Based Collaboration in Information Seeking
}

\author{
Mathias Heilig ${ }^{1}$, Stephan Huber ${ }^{1}$, Jens Gerken ${ }^{1}$, Mischa Demarmels ${ }^{2}$, \\ Katrin Allmendinger ${ }^{3}$, and Harald Reiterer ${ }^{1}$ \\ ${ }^{1}$ Workgroup Human-Computer Interaction, University of Konstanz, \\ Universitätsstrasse 10, 78457 Konstanz, Germany \\ \{mathias.heilig, stephan.huber, jens.gerken, \\ harald.reiterer\}@uni-konstanz.de \\ ${ }^{2}$ Zühlke Engineering AG, Wiesenstrasse 10a, 8952 Schlieren, Switzerland \\ mischa.demarmels@zuehlke.com \\ ${ }^{3}$ Acontrain, Hoheneggstrasse 80, 78464 Konstanz, Germany \\ katrin.allmendinger@acontrain.de
}

\begin{abstract}
Social activities such as collaborative work and group negotiation can be an essential part of information seeking processes. However, they are not sufficiently supported by today's information systems as they focus on individual users working with PCs. Reality-based UIs with their increased emphasis on social, tangible, and surface computing have the potential to tackle this problem. By blending characteristics of real-world interaction and social qualities with the advantages of virtual computer systems, they inherently change the possibilities for collaboration, but until now this phenomenon has not been explored sufficiently. Therefore, this paper presents an experimental user study that aims at clarifying the impact such reality-based UIs and its characteristics have on collaborative information seeking processes. Two different UIs have been developed for the purpose of this study. One is based on an interactive multi-touch tabletop in combination with on-screen tangibles, therefore qualifying as a reality-based UI, while the other interface uses three synchronized PCs each controlled by keyboard and mouse. A comparative user study with 75 participants in groups of three was carried out to observe fundamental information seeking tasks for co-located collaboration. The study shows essential differences of emerging group behavior, especially in terms of role perception and seeking strategies depending on the two different UIs.
\end{abstract}

Keywords: Collaboration, Tabletop, Tangible User Interface, Information Seeking, User Study.

\section{Introduction}

\subsection{Motivation}

Information seeking activities such as searching the web or browsing a media library are often considered as solitary experiences. However, a lot of theoretical and 
empirical work expresses the importance of collaborative activities during information seeking processes. Morris and Teevan [14] give some figurative examples: students working together to complete assignments, friends seeking information about joint entertainment opportunities, family members jointly planning a vacation travel or colleagues conducting research for their projects. Furthermore, Kuhlthau [10] defines information seeking as a constructive process, where social and collaborative activities are essential to advance the knowledge work process.

Working collaboratively enhances evidently the quality of information seeking activities in many different aspects. One example is the increasing coverage of the relevant information space as well as a reduction of unnecessary and redundant work. Another example is the higher confidence in the quality of findings, through the constructive development of strategies and answers in a group, which is often composed of people with different expertise.

\subsection{Reality-Based User Interfaces}

In today's digital information seeking systems collaborative search is not sufficiently supported. One obvious reason is the limitation of desktop or terminal PCs, which are controlled by mouse and keyboard and therefore do not offer appropriate mechanisms for collaborative work. To overcome this gap several researchers (e.g. [2], [13]) have proposed the use of multi-touch tabletops for co-located, collaborative information seeking activities. These researchers assume that the horizontal form factor of a tabletop interface democratizes the interaction between multiple users through the possibilities of simultaneous touch operations. Furthermore, these settings promise a more natural interaction between users in a way that enhances the perception of the others' interaction, gestures and posture during a work and discussion. Additionally, the concept of tangible user interfaces (TUIs) is also proposed as a tool to support collaborative activities [6]. Through the possibility of parallel manipulation and their physical affordance, they are able to further enhance co-located, collaborative activities with digital information systems.

Explanatory models for these effects are often derived from cognitive science and psychology. For instance, the embodiment theory [3], which indicates that our cognitive development is crucially influenced by our physical and social interaction with objects and living beings of our environment, is gaining more and more attention in human-computer interaction. Besides, the field of HCI has started to build up its own explanatory model: the paradigm of reality-based interaction [8] summarizes the findings from cognitive science, the technical evolution with regard to multimodal interaction, and surface, tangible and social computing. The aim of this paradigm is to guide the interaction design of digital systems by putting the emphasis more on the interaction with the real, non-digital world, thus designing it more "reality-based" and more natural. Different input techniques enable multimodal interaction to take advantage of the physical capabilities of the users. To improve the understanding of digital systems, UIs are based on the rules of the physical world. Also the everyday knowledge of the users is regarded as an instrument to design simple and effective computer systems. Reality-based UIs respect the social skills of the users to enable for example collaborative work. Therefore we think that reality-based UIs have the potential to enhance collaborative, co-located information seeking. 


\subsection{Research Focus and Goals}

In this paper we present an experimental user study with 75 participants that aims at clarifying the impact of reality-based UIs and their characteristics on the collaborative information seeking processes in comparison to desktop-based PC UIs. Thereby our focus lies on tightly-coupled collaboration during the exploration and filtering of search results. These tasks are typical situations in information seeking where it is beneficial for people to work together [10]. Reality-based UIs provide adequate features to support this aspect as revealed by several studies (e.g. [5], [12]).

The focus of this work is to detect hidden details on how the interaction, the communication as well as strategies change dependent on different UI types. Special attention of the study was paid to the emerging roles and behavior patterns that occur during collaborative work. Therefore, our study is guided by three research questions to disclose the mechanics of co-located, collaborative information seeking with reality-based UIs: (Q1) How do reality-based UIs influence the interaction strategies in comparison to PC-based UIs? (Q2) What impact do the two UIs types (realitybased and PC-based) have on the communication (verbal and non-verbal) during the group work? (Q3) Are there differences in the occurrence of roles people adopt during the group work depending on the UI?

The paper is composed of six chapters: After the introduction (1) important related work is discussed (2). In this section research is presented that empirically explored impacts of reality-based UIs in collaborative conditions. The insights of these projects will be summarized and the need for our study will be emphasized. Then, the concepts behind the two UIs (3) that were used in our comparative user study will be described. Thereafter, the design of the study and data analysis will be explained (4), followed by the presentation of the results (5). A special focus will be laid on the explanation of different roles and strategies participants adopted depending on the interface condition. Finally, the paper closes with a discussion and a conclusion (6).

\section{Related Work}

In the last years numerous systems have been developed that explicitly or implicitly adapt reality-based concepts. Many of these approaches have been designed to enhance co-located collaborative work. The following section introduces selected approaches that focus not only on the design of such UIs, but also on the evaluation showing their potential practical impact.

\subsection{Interactive Surfaces}

The interactive surface WeSearch [13] has been designed for collaborative web search to leverage the benefits of tabletop displays for face-to-face collaboration. The system was also an integral part of a user study, which showed that tabletop displays are effective platforms to facilitate collaborative web search. Furthermore, the study revealed that tabletop displays enhance the awareness of group members' actions and artifacts such as search criteria and allow natural transitions between tightly- and loosely-coupled work styles. 
Another research project [11] evaluated how different configurations of input (single-mouse, single-touch, multi-mouse and multi-touch) influence the balance of inter-user participation around a tabletop interface during planning tasks. The project showed that tabletop UIs could be designed to enable a more balanced participation. The paper further reported that with touch interaction fingers rather than voices do the talking: "interactive participation is more equal with touch input and multiple entry points than with mice or single input, but verbal participation is not".

Isenberg et al. [7] investigated co-located collaboration on a multi-touch tabletop for complex visual analytics. They intensively analyzed the closeness of teams' collaboration and the influence of the group work on the task performance. The study showed that teams, which worked tightly together, were more successful completing the task and required fewer support. Furthermore, the study presented eight types of collaboration styles that identify how people work together while solving problems.

\subsection{TUIs and Hybrid Interactive Surfaces}

TUIs are also proposed as a tool to enhance co-located collaboration [6]. They benefit from possibilities such as parallel, physical manipulation and further inherit implicit characteristics such as a better awareness of the others' actions through their visibility and their physical affordance. This promises to facilitate the involvement and active participation in group work without explicit verbal communication. The use of hybrid interactive surfaces - tangible interaction in combination with tabletop displays [18] is an approach to combine the advantages of interactive surfaces and TUIs for colocated collaborative work. However, until now there are only few empirical studies that explored the impact of hybrid interactive surfaces.

The user study in [4] showed first results that hybrid interactive surfaces exhibit considerable advantages, with respect to parallel interaction of multiple users, in comparison to classic search interfaces. Furthermore, Jetter et al. [9] introduced with their Facet-Streams a hybrid interactive surface for co-located, collaborative product search. The system uses techniques of information visualization with tangible and multi-touch interaction to materialize collaborative search on an interactive surface. Two user studies demonstrated the potential of this hybrid UI concerning visual and physical affordance as well as simplicity in interaction. With regard to collaborative work the authors observed an increased awareness and better mutual support among collaborators and seamless transitions between tightly-coupled collaboration and loosely-coupled parallel work.

\subsection{Insights and Open Research Areas}

The discussed research projects reveal that multi-touch tabletop displays offer promising possibilities for co-located, collaborative work such as the equal access to information, the smooth transitions between individual and collaborative activities as well as providing a more balanced participation. Besides, hybrid interactive surfaces show additional qualities for collaboration such as parallel, physical manipulation or the increased awareness and better mutual support among collaborators.

However, until now the influence of such UIs on collaborative work, especially on information seeking, has not been explored in detail and efforts to identify the 
mechanics of collaboration in these tasks and how reality-based UIs might support them are missing. Therefore, the aim of our experimental user study is to provide detailed insights on whether and how the interaction, the communication as well as strategies of users during collaborative exploration and seeking activities changes dependent on different interface types. Since the introduced studies already revealed that reality-based UIs might offer benefits beyond efficiency and result quality [5], these parameters are not the main focus of our study. Instead, our findings will be manifested in different behavior pattern and roles that occur during group work in information seeking.

\section{Experimental User Interfaces}

In order to be able to study the influence of reality-based UIs on collaborative search, we designed two UI configurations (see fig. 1). Each UI represents one specific interface type (reality-based versus PC-based). The experimental systems differ only in the interaction mechanics (independent variable), whereas search as a shared and co-located experience should remain stable to assure a fair and valid comparison. The following sections introduce the shared characteristics of both UIs.
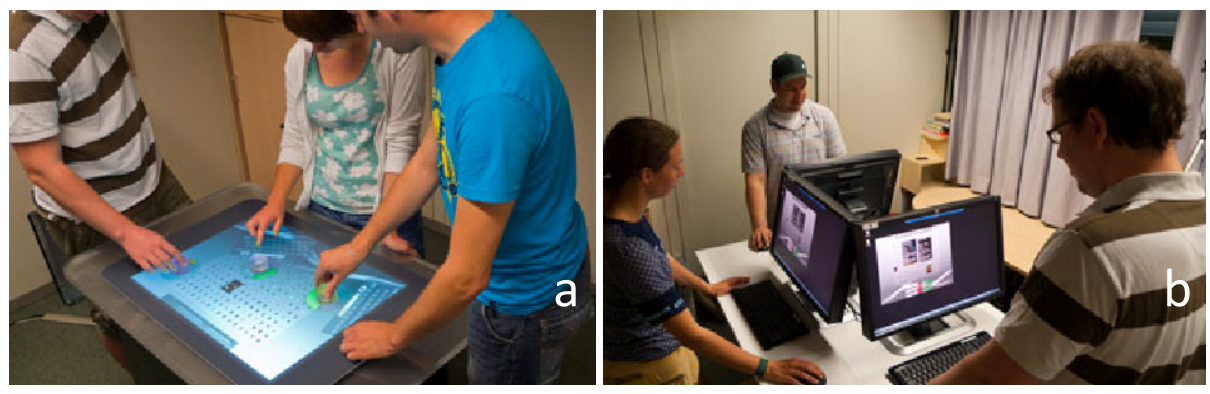

Fig. 1. (a) The Search Token as reality-based UI; (b) Three Synchronized PCs as alternative UI

\subsection{Interaction and Visualization Principles}

Data Collection and Visualization. We designed a visualization that arranges about 200 movie objects in a grid-structured canvas (see fig. 2a). In the default view the movie objects are displayed as poster representations. Semantic zooming was used to display three different levels of detail for each object (see fig. 2b). Each UI type provides the possibility to enter filter keywords, which trigger the semantic zoom of the matching information objects. All objects can freely be arranged on the canvas by dragging operations (either with touch or by mouse). Users are thus able to create personal clusters of intermediate search results for discussion in the group.

Dynamic Query and Sensitivity. One important concept of the two UIs is based on dynamic filter mechanisms that bring interesting information objects to the users' attention using keyword-based dynamic queries [1]. To support multiple users during collaborative work in a co-located environment it is crucial that all actions of other 
group members are accessible and can be comprehended by everyone involved in the information-seeking process. To address this issue, a filter method inspired by the concept of sensitivity [15] is used for our UIs, which is in contrast to common filter strategies that instantly hide all non-matching objects upon filtering. To express that a specific information object matches a user defined filter criterion the visual representation of this object is enlarged, emphasizing its importance to all collaborators. All information objects that do not match the filter query are decreased in size and transparency, allowing the user easily to visually distinguish between matching and non-matching information.
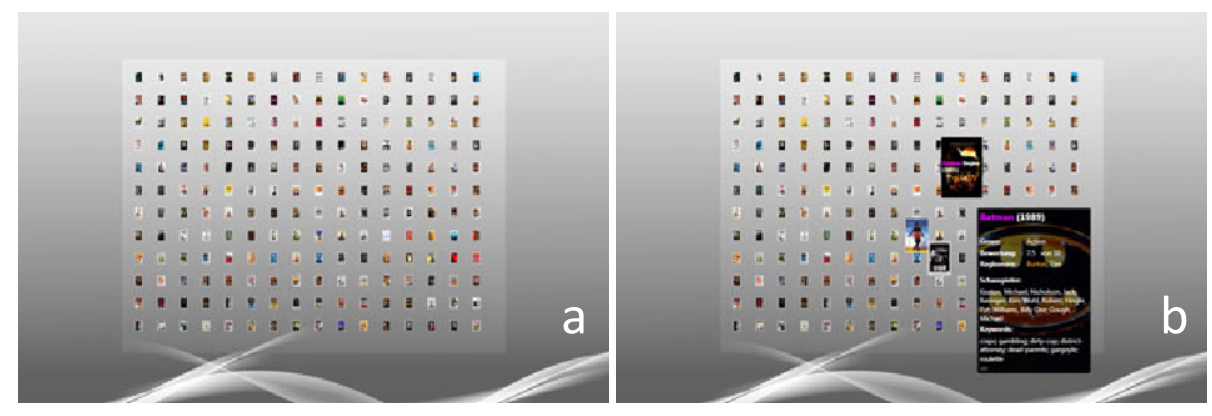

Fig. 2. (a) The visualization with about 200 media objects. (b) Matching media objects increase in their size and offer three semantic zoom levels representing different levels of detail.

The combination of multiple filter criteria is a fundamental concept to enable all collaborators to personally get involved in the search and exploration process. In the presented interfaces users are able to combine multiple filter criteria using Boolean operations. Following the concept of sensitivity described above, information objects that match more than one filter criterion are represented even bigger than the ones that only match a single filter criterion. The default operation that is used to combine the different filter criteria is a Boolean AND. Additionally, the users can interactively alter the weight of all filter criteria. While this allows much more powerful search operations, it can also enhance the collaborative process in the way that a collaborator is able to scale the weight of a specific criterion up or down to better communicate the corresponding aspects to the other collaborators. The mathematical model behind the weighting of the filter criteria is based on the concept of weighted Boolean [17].

In addition, a color highlighting mechanism visually links matching information objects with the corresponding filter criterion (see fig. 3). Each criterion has a distinct color, which is also used to highlight a matching keyword in the detail information of an object. The colors in the result view can be used to associate the important information objects with the user that is manipulating the corresponding filter criterion as proposed with the concept of collaborative brushing and linking [7].

Resize Algorithm. The resize algorithm (see fig. 3) applied in the two UIs is based on a simple mechanism [4]: Each filter consists of a keyword and a weight. The keyword can either match or not match a specific information object (when it is found or not in the object's metadata). The weight of each filter can be between 0 and 2 and corresponds to 
the resize factor this filter will add to the size of a matching information object. Therefore, a weight between 0 and 1 will shrink all matching information objects, which corresponds to a (weighted) Boolean NOT operator. A weight between 1 and 2 will increase the size of all matching information objects. For example, a filter that matches a specific information object and has a weight of 0.1 , would shrink this object to a tenth of its size, where as a weight of 1.6 would increase its size by $60 \%$.

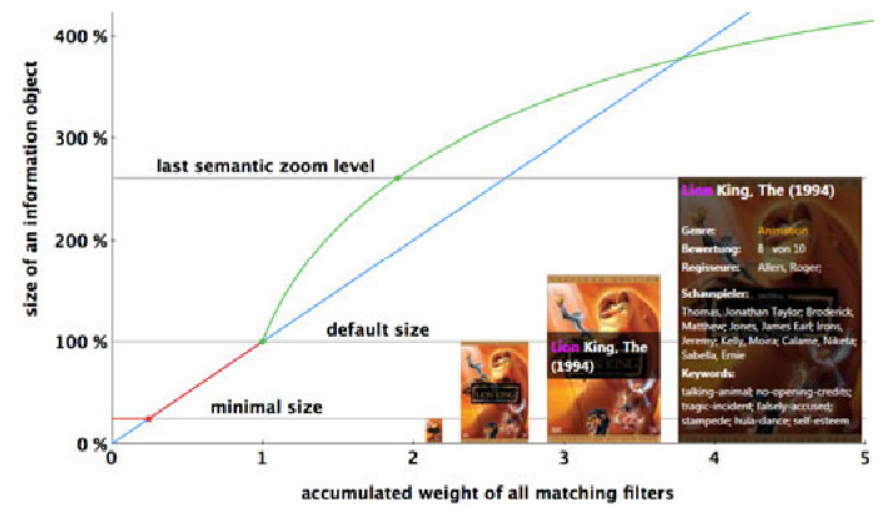

Fig. 3. Schematic diagram of the resize algorithm for media objects: The linear function (blue) is altered by a logarithmic correction (green) to enhance the objects' zooming behavior

The real size of an information object is calculated out of all weights of every matching filter. For example if three different filters match an information object, one with a weight of 2 , one with a weight of 1.5 and one with a weight of 0.8 , the size of this object will be 2.4 times $(2 * 1.5 * 0.8=2.4)$ of its default size. This simple algorithm is modified through the application of an logarithmic function (see fig. 3 , green line) to help to solve two different problems: (1) Information objects that match a filter should get bigger even with low weights, so that more detail information can be shown early; (2) Once all detail information of an object is visible, the growth of this object should be damped so that it does not cover too much screen space.

\subsection{Reality-Based User Interface: The Search Token}

The foundation of the Search Token as the first UI condition for the experiment is built upon a physical object that can be placed on a multi-touch tabletop display (see fig. 1a). The Search Token as hybrid interactive surface enhances the visibility of interaction with the system since their physical appearance provides a higher visual and tangible affordance than a UI that is solely based on digital sliders, text fields, buttons, etc.

Similar to the Parameter Bars [16] a Search Token can be dynamically configured with different search parameters, thereby acting as filter to the information space. By placing a Search Token on the tabletop, it is augmented by a visualization. One Search Token consists of four main parts: the transparent Plexiglas cylinder (the physical object), the textbox for the filter keywords, the circular indicator for the weight of the entered search criterion and the virtual on-screen keyboard (see fig. 4a). This keyboard can be temporarily hidden to save screen space. The visualization is 
virtually connected to the physical token like a digital shadow, following its movement on the screen. Moving and turning a token thus enables all participants around the tabletop to access the token's visualization. When a search criterion is entered, rotating the Search Token allows users to define the criterion's weight. Thereby, the circular indicator around the physical cylinder interactively shows the adjusted weight and the Plexiglas cylinder glows in the highlighting color (see fig. 4b). To combine several search criteria, multiple Search Tokens (three in the user study) can be used on the surface of the tabletop display.

With regard to reality-based UIs [8], the Search Token qualifies as such as it incorporates main characteristics in a comprehensive manner: the physicality of people and objects, the social context as well as the environment.
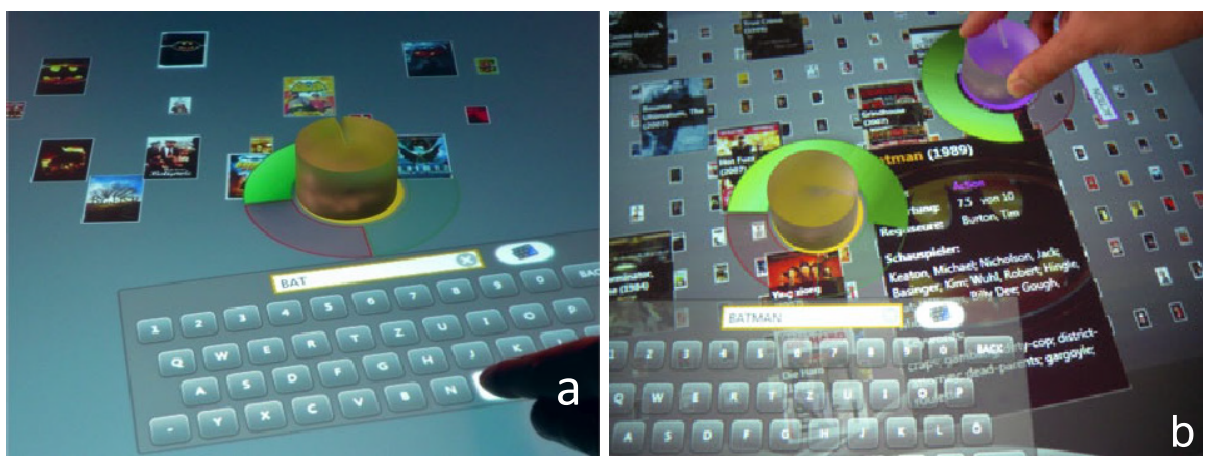

Fig. 4. (a) A Search Token as hybrid surface on a multi-touch tabletop display enable users to simultaneously enter search criteria via on-screen keyboards; (b) By turning a Search Token, the weight of a search criterion can be adjusted

\subsection{Synchronized PC User Interface}

In contrast to the Search Token, we designed the second UI condition intentionally against the principles of reality-based interaction. However, as we wanted to especially focus on the mechanics of reality-based interaction, certain aspects should remain stable. This includes the co-located setting as well as the possibility for parallel interaction. Therefore, three PC-devices were triangularly arranged on a table that had a similar size as the multi-touch tabletop (see fig. 1b). Through a "real-time" synchronization of the visualization and filters on all clients, the participants share one logical view on the UI (see fig. 5a). Via the text boxes in the lower area of the screen the participants are able to simultaneously define search criteria (see fig. 5b). The sliders next to the search boxes allow defining the weight of the search criteria.

This setting enables each collaborator to equally participate in the interaction through the use of mouse and keyboard belonging to the particular PC. With this setting the participants had the possibilities to see the faces, gestures and posture of the group members, to communicate with each other, and to interact simultaneously, comparable to the reality-based UI. Basically, the differences between the UIs are the following: (1) the form factor, (2) the physically merged interaction space, and (3) the interaction (mouse/keyboard versus Search Token and touch interaction). This allows us to isolate the mechanics of interaction (IV) for our experimental user study. 

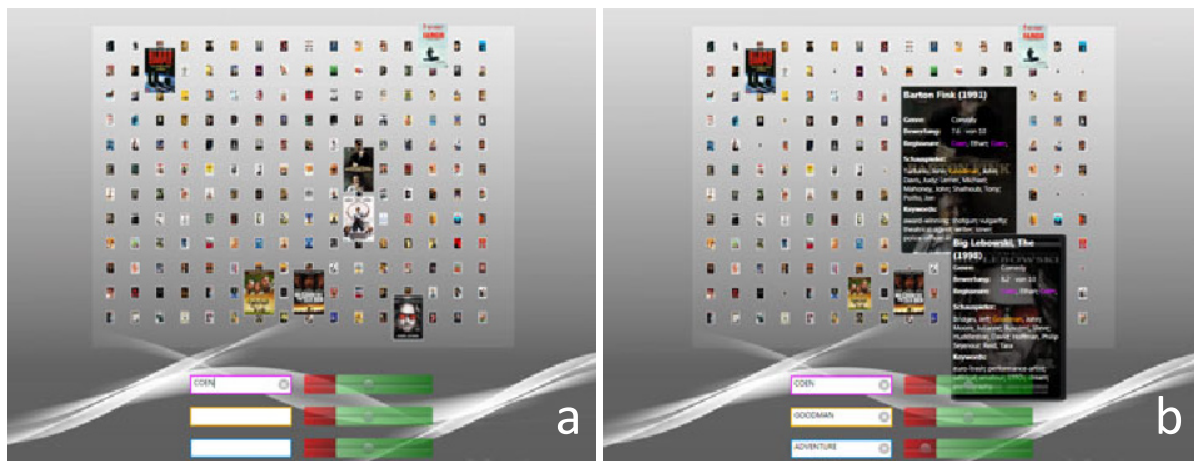

Fig. 5. (a) Participants share a synchronized logical view from different PCs and are able to simultaneously enter search criteria into the text boxes; (b) Via the slider widgets that are assigned to the text boxes, it is possible to adjust the weight of search criteria

\section{Experimental User Study}

Our experimental user study was intended to explore in detail, how collaboration and peoples' behavior in the context of information seeking would be affected by using either reality-based UIs or PC-based UIs. Figure 1 shows the setup of our experimental interfaces, as discussed in the previous chapter.

\subsection{Participants and Design}

We used a between-subjects design (IV: UI, reality-based vs. PC) with 75 participants, who were randomly assigned to 25 groups of three (triads, 12 tabletop and 13 PC groups). Participants were students or university faculty (39 females and 36 males) from a variety of non-technical institutes. The average age was 26 (SD = 7.4 years). Triads are a typical setting for small groups working together on an information seeking assignment and our participants stated that they were familiar with such situations. Information seeking was also a very frequent task amongst them. Furthermore, nearly all of our participants had prior experience with using touch displays (e.g. smart phones). We decided to apply a between-subjects design as we identified several aspects that can have a significant and uncontrollable influence on the results of a within-subjects design: First, the novelty of a tabletop UI with tangibles might evoke a strong "wow"-effect and lead to a bias when putting the reality-based UI configuration in contrast to the PC-based UI. Second, group dynamics evolve over time as people get to know each other. Third, in within-subjects designs some participants tend to transfer strategies of the first UI to the following. Even a counter-balanced within-subjects design might not have been able to rule out such interaction effects. We also explicitly decided to divide participants into groups in which they did not know each other. While this may not reflect a real-world situation, it allows us a better level of control of inter-person relationships and their possible effects on group dynamics. 


\subsection{Procedure and Tasks}

After a short introduction, each group was given a five minute instruction to the respective UI followed by a five minutes free exploration phase to get to know the system and each other, before working collaboratively on a total of four tasks, two of them being training tasks. These training tasks required the participants to search for a movie object within the collection that matches various attributes (e.g. genre: "romance" or keyword: "murder"). Subsequently, the two "real" tasks were designed in a way to simulate a realistic negotiation situation in which compromises need to be made. They required participants to agree on a movie object within a limited amount of time. For each task every participant received one criterion during the first task or two criteria during the second task (e.g. keyword: "explosion", genre: "crime") representing his or her fictive interests. In order to simulate a realistic collaborative situation, the tasks were designed in a way that made it impossible for the group to satisfy the total of all criteria. Thus, all participants had to negotiate whose personal criteria to minimize (e.g. by reducing the weight for one or several criteria) or whose to give up completely. We analyzed the quality of these compromises by means of the results' distance to the non-existing ideal compromise. However, the compromises' quality didn't differ significantly between the two conditions. A time limit of 5 minutes per task was used to control the session duration and increase participants' motivation to come to a decision. We did not interrupt users before a final decision was made, since the time limit was not intended as a sharp criterion for the completion of a task. The mean duration of these tasks was 4:46 minutes (mean $=286 \mathrm{~s}, \mathrm{SD}=74 \mathrm{~s})$. After completing the tasks, each participant filled out a personal questionnaire about their subjective assessment of the group work and the UI. Overall, the whole session per group took about 45 minutes and participants were compensated with 15 EUR.

\subsection{Data Collection and Analysis}

We used a variety of data collection techniques including questionnaires (pre- and posttest), interaction logging as well as video and audio recording. Two video streams captured both the detailed interaction on the display and the overall group dynamics from a bird-eye view perspective. In case of the PC interface, a screen capture recording was used to show the detailed interaction on all displays. Each participant wore colored batches and bracelets, allowing us to easily allocate their interactions. Video and screen recordings were analyzed in detail. Based on several overall screenings, a complex coding scheme was developed, which focused on three aspects: (1) the individual interaction of participants with the system (e.g. typing in a keyword, moving an object), (2) the visual focus and attention of participants either to the system or amongst another (e.g. turning around, looking up), and (3) the kind of verbal communication between participants. The coding scheme was then applied to the last and most complex task with two given criteria for each participant. As coding the data in this depth is impossible within one session, we applied a multi-pass coding, with each pass focusing on one of the three aspects. To ensure inter-coder reliability, the material of 4 groups (two of each UI configuration) was encoded a second time by a researcher not involved in the study (statistically analyzed with Cohen's Kappa, $\kappa=0.67$ ). Additionally, we looked for interesting patterns and captured such scenes during the coding sessions. 


\section{Study Results}

In this section we describe, how the major interaction strategies, communication behavior and roles the participants adopted to solve the tasks differ between the two UI conditions. In the PC condition, each participant was in theory able to work with an individual PC. However, we did not enforce participants to spread around the individual PCs. Interestingly, two groups gave up the advantage to work simultaneously and shared one PC. Upon closer inspection, it became obvious that in these groups one participant took on a dominant role and mainly solved the tasks while the other group members showed a very cautious behavior. To allow a detailed and reasonable comparison between the interface conditions, we excluded these groups, leaving us 23 triads (69 participants, 12 Search Token and 11 synchronized PC groups). T-tests were used to analyze the data for statistical differences.

\subsection{Interaction Strategy}

Simultaneous Interaction. Regarding our first research question (Q1, interaction strategies) we were interested in how often people interact in parallel. This aspect is reported as one major advantage of reality-based UIs (e.g. [6]). To compare the two conditions, we use percentages of task time as a normalized value. The results show that the reality-based Search Token condition featured more simultaneous interaction than the PC-based condition (see fig. 6). In 15.3\% (SD = 4.97\%) of the time all three participants interacted in parallel. In comparison, the PC-based condition showed this behavior in $3.45 \%(\mathrm{SD}=1.22 \%)$ of the time, with the difference between the conditions being statistically significant $(\mathrm{t}(23)=1.78, \mathrm{p}=0.04$; statistically analyzed on group level). Parallel interaction of two participants occurred with the Search Token condition in $47.3 \%(\mathrm{SD}=39.11 \%)$ of the time and happened therefore significantly more often $(\mathrm{t}(23)=2.16, \mathrm{p}=0.004)$ than the synchronized PC UI $(11.9 \%, \mathrm{SD}=8.01 \%)$.

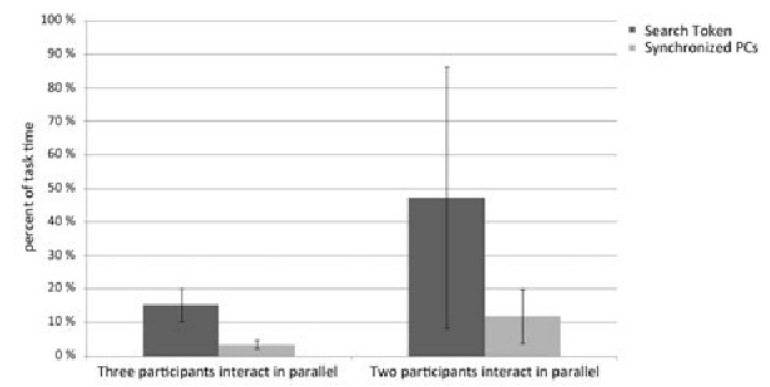

Fig. 6. The diagram shows the time (in percent of the total task time), when three participants and two participants work simultaneously with the system

Interface-Element Sharing. We identified an interesting behavior of some participants in the Search Token condition. Without being asked, they occupied a Search Token, which was previously in use by another group member (see fig. 7). 
Such a behavior never occurred in the PC-based condition (No participant used a text box or slider, which was already in used by another group member). This suggests that the threshold to intervene the interaction of others is lower with the Search Token than with the PC-based UI. We observed that such a behavior pushes the collaboration through a closer and mutual interaction: Once executed, the other participants imitated this behavior and also used Search Tokens of other participants on the tabletop display. However, in one case such "token-takeover" led to the other participants backing out and interacting less often with the system.
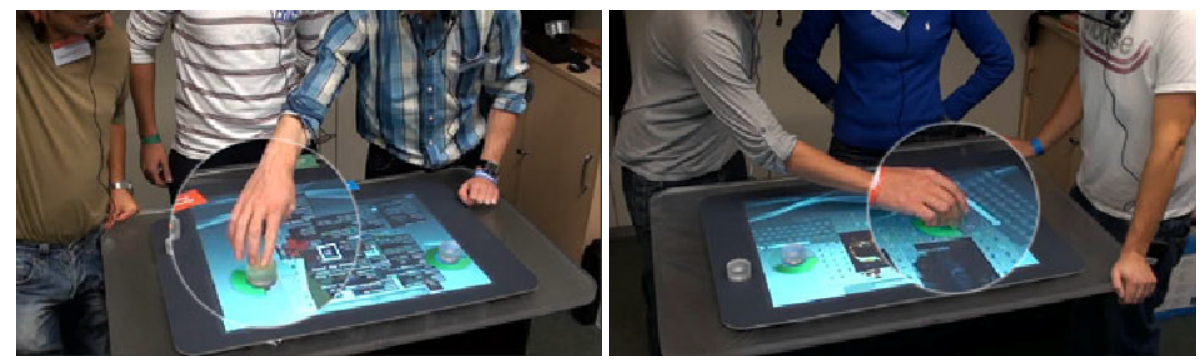

Fig. 7. The figure shows two exemplary scenes, where a participant is taking over a Search Token from another group member

\subsection{Communication}

Verbal Communication. The second research question (Q2) asked for the impact of communication. We classified communication into process-dependent (strategic meta-contributions to proceed the task, e.g. "let's sort these movie objects to the right!" or "I take the upper search box"), task-dependent (contributions to solve the task with regards to content, e.g. "Do you think 'Gladiator' is a biography" or "Is 'American History X' a cruel movie?"), no communication, and undefined communication. However, analysis showed no significant differences between the groups in terms of the different types of communication. For example, the triads in the Search Token condition featured 'no communication' in $14.63 \%$ (SD $=3.97 \%$ ) of the time. In comparison to the triads in the PC-based condition $(16.32 \%, \mathrm{SD}=4.47 \%)$ we detected no significant difference $(\mathrm{t}(23)=2.08, \mathrm{p}=0.84)$. A deeper analysis with process-dependent, task-dependent communication, and undefined communication variables also revealed no significant differences.

Non-verbal Communication. An important aspect of non-verbal communication is the visual focus of the participants during the group work as an indicator for attention. In both UIs the visual focus laid to a great extent on the display(s) of the system. The duration all three participants collectively having their visual focus on the system was $92.68 \%$ ( $\mathrm{SD}=21.34 \%$ ) of the time in the Search Token condition. The groups in the synchronized PC condition shared their visual focus on the system's display in $80.66 \%(\mathrm{SD}=24.58)$ of the time $(\mathrm{t}(23)=2.08, \mathrm{p}=0.054)$. By further analyzing the video material for this phenomenon we detected that with the Search Token UI gestures and posture were perceived from the other group members without needing 
to look up from the display. Whereas the participants in the PC condition had a lot of short interruptions in order to see and perceive the non-verbal expressions of the other group members. Furthermore, we noticed that several participants in the Search Token condition unconsciously used non-verbal activities to communicate involvement and active participation. They for example expressively held a Search Token (see fig. 8) and thus showed the other group members that they took part in the group work.
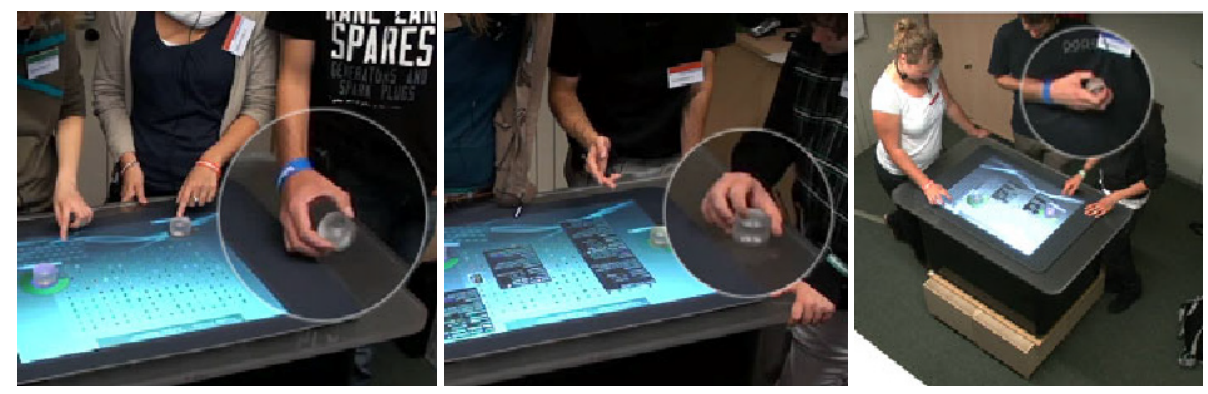

Fig. 8. The images show three examples of participants, which hold a Search Token in their hand without using it for interaction, but for communicating involvement

\subsection{Roles of Collaboration}

Profiling. While chapter 5.1 and 5.2 elaborated on the differences between the UI conditions on a group level, we were also interested to see, if participants adopt different roles depending on the UI condition (Q3). For analysis, we generated a quantitative profile for each participant based on the encoded video material. This profile was composed of the same three dimensions as analyzed on group level, with (1) system interaction (2) visual attention and (3) verbal communication. The system interaction was subdivided into the behaviors: no interaction, filter action, and object manipulation and allowed us to get an understanding what type of interaction a person prefers. Visual attention is decomposed into no attention, attention to the system, and attention to other team members. Verbal communication is separated into the behaviors: no communication, process-dependent communication, task-dependent communication and undefined communication. The time in percent a participant showed one of the behaviors during the task session was plotted on the axis of a spider-gram. To recognize similar profiles, we printed out the profiles of the 69 remaining participants and asked two different experimenters to sort them independently into clusters after visual similar behavior pattern and without being aware of which plot belonged to which of the two interface configurations.

Later on, we took the intersection of these clusters and analyzed the behavior of the participants in the video material. Participants that showed a behavior that did not fit to the other participants of a cluster were excluded (This happened only in 2 cases). We decided that a cluster had to contain a minimum of 6 participants to be regarded as a role (that corresponds to a probability of about $25 \%$ that a role occurred in one of the 23 
groups). This way we extracted 5 roles (see fig. 9) with 39 of our 69 participants $(56.5 \%$ ). In the following, we present the key characteristics of each role. Interestingly, most roles can be used to easily distinguish between the two interface conditions. Thereby, this allows us to point out in detail how the interfaces affected peoples' role behaviors.
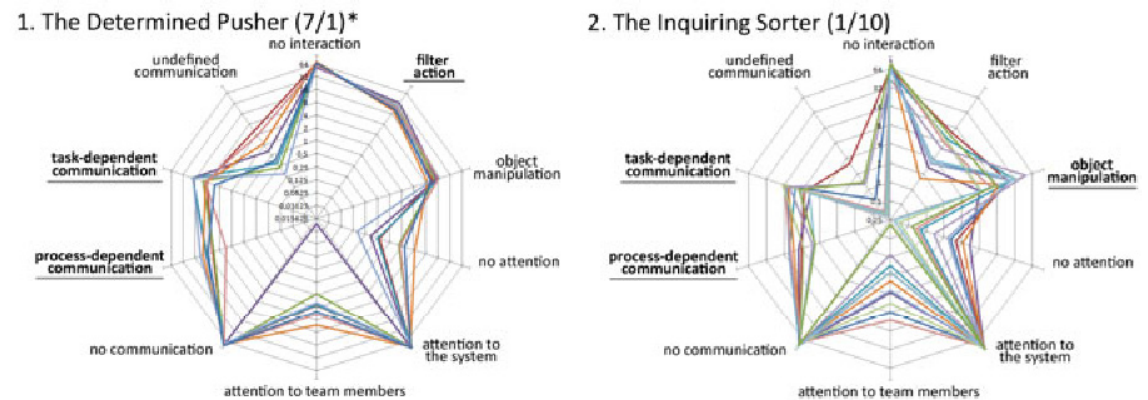

3. The Cautious Attendee (2/4)
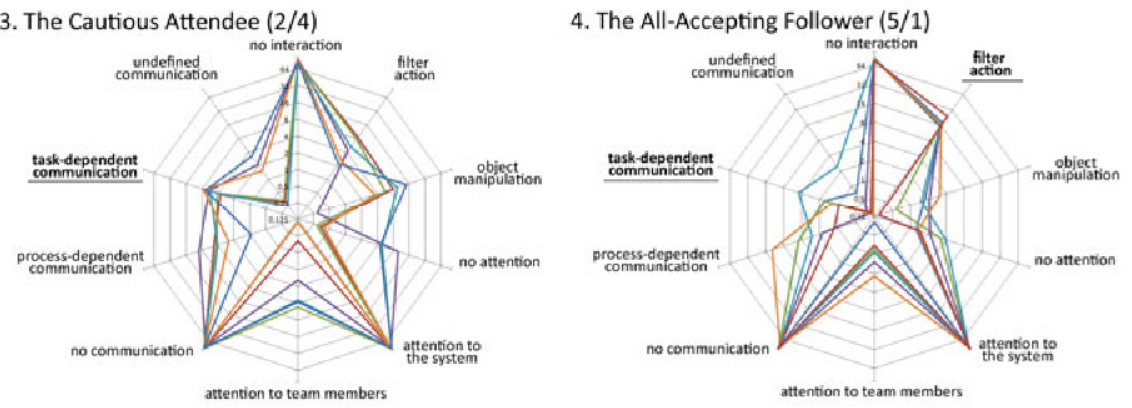

5. The Interested Observer $(8 / 0)$

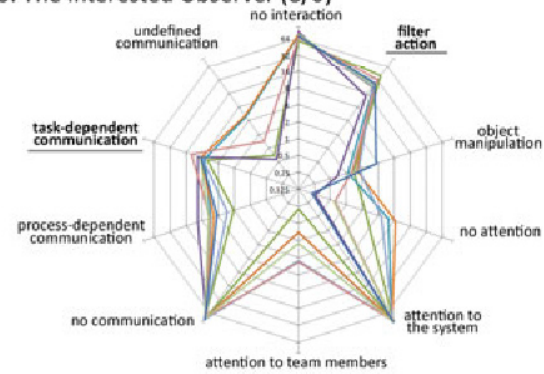

* The values in brackets after the role names shaw the distribution of this role (Search Token/PC-based Condition).

The logarithmic-scaled spider-gram axes show the time in percent used from a single participant during the task session for a special behavior (e.g. filter action, object manipulation). The detected behavior patterns are highlighted through bold and underlined labels onthe axes.

Fig. 9. The spider-grams show the profiles of the five different roles that participants adopted during the group work

Overall with regard to solving the tasks we could distinguish between participants taking on a lead role and participants taking rather a cautious or passive role. What is interesting is that participants impersonated these roles differently depending on the interface configurations. We will first discuss the active/lead participants (role $1 \& 2$ ) and then continue with the more passive/cautious roles (role 3, 4, 5). 


\section{Leading and Active Participants}

Role 1: The Determined Pusher. The behavior of this role was adopted by at least one person in 6 (out of 12) groups in the Search Token condition but only one group (out of 11) in the PC-based condition. The determined pusher is a very active participant and tries to engage the other team member to work together and to solve the task. Further, the participant is very attentive and frequently contributes verbally task-dependent (e.g. "Let's inspect the movie Gladiator"), but also strategic (processdependent, e.g. "I propose to delete all criteria!"). This role features a lot of filter actions to communicate own ideas how to solve the task. However, the participant involves the other group members as well through discussion, gestures, and in case of the Search Token condition, through the sharing of Search Tokens (see chapter 5.1, Interface-Element Sharing).

Role 2: The Inquiring Sorter. The counter-part to the determined pusher is the inquiring sorter. In 7 groups of the PC-condition at least one person adopted this role while this happened only once in the Search Token condition. Similar to the determined pusher, these participants try to animate the other team members to actively take part in group work through intensive and motivating feedback on verbal contributions and actions of other group members. However, and contrary to the determined pusher, the interaction with the system did not focus on filter activities. Instead the inquiring sorter interacted with the virtual media objects in the visualization (e.g. sort the objects matching after search criteria) to highlight special correlations in the collection. As discussed in the "interface-element sharing" incident, these participants also did not take over the search boxes from other participants.

Discussion. We conclude that the Search Token condition allowed active lead users to take on a more dominant role within the groups. They took the chance to influence or even control the strategy, the interaction (by controlling the physical tokens and filter keywords directly) and also the overall group participation (by handing over tokens or using tokens to communicate). In contrast, such participants in the PC condition seemed to be limited in their influence on the system interaction and mainly focused on highlighting search results and sorting in order to take on the lead role.

We assume that one important reason for this phenomenon is that the reality-based interface is shared in its physical entirety. Thereby, conflicting activities (e.g. two persons reaching for the same token) can be easily resolved by accepted and wellestablished social protocols. The shared, but virtual PC condition makes resolving such conflicts much more difficult. For example it can easily happen that two persons try to interact with the same search box at the same time. The physical awareness of the others' actions is missing, which leads to conflicting interactions. Therefore, we think that dominant persons avoided these conflicts and thereby had fewer possibilities to influence the group activities. However, one has to be aware of that dominant persons in a reality-based condition can also have a potentially larger negative impact on the group. 


\section{Cautious and Passive Participants}

Role 3: The Cautious Attendee. This role emerged mainly in groups, which were in the PC condition (4 groups compared to 2 groups in the Search Token condition). Participants that adopted this role can be characterized as persons easily conceding to strategy decisions of other group members. While they take part in task-dependent communication, they often abandon own ideas and mainly say something to support the decision and interaction of other group members (e.g. "That's right.", "Yes, these are the two movies"). From an interaction point of view, they only engage with the system during the initial phase when every group member enters their keywords, but stay passive during the refinement and consolidation phase.

Role 4: The All-Accepting Follower. The counter-part to the cautious attendee adapted the role of an "all-accepting follower". This role, which emerged in 4 groups of the Search Token UI and only once with the synchronized PC UI, is similarly characterized by an incessant acceptance and agreement on the strategies of the other group members. While these participants seem even more cautious in verbal communication (e.g. "Yes, that's my opinion, too.", "This one is also a movie with a murder, right?"), they did use the Search Tokens to interact with the system. This happened most of the time in parallel with another team member, following the lead of this person.

Role 5: The Interested Observer. Interestingly, while sharing some of the characteristics of the cautious attendee and the all-accepting follower, we could identify an additional different role within the Search Token condition (6 groups). Most of the time, these participants simply observed the system interactions of other group members in a very interested manner (especially during the refinement phase with sorting and arranging of objects). Interaction occurred mostly in the early phases through pre-decided filter actions and with a Search Token they had placed by themselves on the tabletop. However, in later phases, while they actively participated in group work through task-dependent verbal contributions (e.g. "What movie did we have earlier?", "That is an action movie!", "No, this movie doesn't match our criteria!"), they left the execution of proposed strategies to the other group members.

Discussion. We conclude that the cautious/passive participants in the reality-based condition capitalized on a broader variety of means of expression to take part in the group activities. On the one hand, as discussed in section 5.2 about non-verbal communication, they were able to show their involvement in the group activities by postures, gestures, and just holding a token. On the other hand, they were much more active regarding filtering the information space by using the tokens, even in later phases. In contrast, such participants in the PC condition were only active in the beginning of a task and then seemed to use the PC monitor as privacy shield, allowing them to stay passive without having to fear any consequences. However, cautious participants in the reality-based condition were also more in danger of being pushed out of the group. As discussed in section 5.1, dominant participants sometimes took over the Search Token of others. In a few of these cases the now "tokenless" and cautious participants retired their selves from the collaboration completely. 


\section{Conclusion}

We presented an extensive experimental user study that provides a rich understanding of the influence different interface types (reality-based versus PC-based) can have in a collaborative information seeking situation. With respect to our research question Q1 (interaction strategies) we conclude that participants working with the reality-based UI, developed a wider variety of information seeking strategies such as interfaceelement sharing or simultaneous interaction compared to the participants in the PC condition. We assume that this is caused by the natural and "materialized" interaction and consequential qualities (e.g. physical awareness and manipulation). Concerning our second research question $(\mathrm{Q} 2$ : communication) we identified mixed findings: As we analyzed the groups' verbal communication, we didn't determine noticeable differences between the two interface conditions. These results fit very well to the findings of Marshall et al. [11], who showed that verbal participation in group work is not constrained by the type of input. However, regarding non-verbal communication, we observed that in contrast to the PC condition the participants in the reality-based condition seamlessly perceived gestures of their group members and used the physical artifacts to communicate and produce meaning. We further detected five different roles (Q3: roles of collaboration), which allow to easily distinguish between the two interface conditions. Participants, although having similar personalities (e.g. dominant/active persons or cautious/passive persons) often adopted different roles depending on the interface condition they were using. One determining factor for this phenomenon was the emerging social environment triggered by the reality-based UI. Another one was the apparent, multi-faceted possibility to physically express and communicate ideas through tangible interface elements (e.g. interface-element sharing). During the user study some interesting alternative set-ups of our experiment with respect to the PC condition came up that would be beneficial to deepen the insights of our findings and would disclose further research questions: 1) One shared PC with a single large display, that can be controlled by multiple keyboards and mice; 2) the participants around the table will be equipped with individual tablet PCs that share a synchronized view and can be controlled by touch input. Altogether, this paper demonstrates that the application of reality-based interfaces tremendously alters the behavior of collaborators in small groups across multiple dimensions.

\section{References}

1. Ahlberg, C., Williamson, C., Shneiderman, B.: Dynamic queries for information exploration. In: CHI 1992, pp. 619-626. ACM, New York (1992)

2. Amershi, S., Morris, M.R.: CoSearch: A System for Co-located Collaborative Web Search. In: CHI 2008, pp. 1647-1656 (2008)

3. Gibbs, R.W.: Embodiment and Cognitive Science. Cambridge University Press, Cambridge (2006)

4. Heilig, M., Demarmels, M., Allmendinger, K., Gerken, J., Reiterer, H.: Fördern realitätsbasierte UIs kollaborative Rechercheaktivitäten? In: Mensch und Computer 2010, pp. 311-320 (September 2010)

5. Hinrichs, U., Carpendale, S., Scott, S.D.: Evaluating the effects of fluid interface components on tabletop collaboration. In: AVI 2006, pp. 27-34 (2006) 
6. Hornecker, E.: Understanding the Benefits of Graspable Interfaces for Cooperative Use. In: Coop 2002, pp. 71-87. IOS Press, Amsterdam (2002)

7. Isenberg, P., Fisher, D., Morris, M.R., Inkpen, K., Czerwinski, M.: An Exploratory Study of Co-located Collaborative Visual Analytics around a Tabletop Display. In: VAST, pp. 179-186. IEEE Computer Society, Los Alamitos (2010)

8. Jacob, R.J.K., Girouard, A., Hirshfield, L.M., et al.: Reality-Based interaction. In: CHI 2008, p. 201. ACM Press, New York (2008)

9. Jetter, H.-C., Gerken, J., Zöllner, M., Reiterer, H., Milic-Frayling, N.: Materializing the Query with Facet-Streams - A Hybrid Surface for Collaborative Search on Tabletops. In: CHI 2011, Vancouver, BC, Canada, May 7-12 (2011)

10. Kuhlthau, C.C.: Seeking meaning: a process approach to library and information service. Libraries Unlimited (2004)

11. Marshall, P., Hornecker, E., Morris, R., Dalton, N.S., Rogers, Y.: When the fingers do the talking: A study of group participation with varying constraints to a tabletop interface. In: 2008 Workshop on Horizontal Interactive Human Computer Systems, pp. 33-40. IEEE, Los Alamitos (2008)

12. Morris, M.R., Paepcke, A., Winograd, T.: TeamSearch: Comparing Techniques for CoPresent Collaborative Search of Digital Media. In: First IEEE International Workshop on Horizontal Interactive Human-Computer Systems (TABLETOP 2006), pp. 97-104. IEEE, Los Alamitos (2006)

13. Morris, M.R., Lombardo, J., Wigdor, D.: WeSearch. In: CSCW 2010, p. 401. ACM Press, New York (2010)

14. Morris, M.R., Teevan, J.: Collaborative Web Search: Who, What, Where, When, and Why. Synthesis Lectures on Information Concepts, Retrieval, and Services 1 1, 1-99 (2009)

15. Tweedie, L., Spence, B., Williams, D., Bhogal, R.: The Attribute Explorer. In: CHI 1994, pp. 435-436. ACM, New York (1994)

16. Ullmer, B., Ishii, H., Jacob, R.J.K.: Tangible Query Interfaces: Physically Constrained Tokens for Manipulating Database Queries. In: INTERACT 2003, pp. 279-286. IOS Press, Amsterdam (2003)

17. Waller, W.G., Kraft, D.H.: A Mathematical Model of a Weighted Boolean Retrieval System. Information Processing \& Management 15 5, 235-245 (1979)

18. Weiss, M., Wagner, J., Jansen, Y., Jennings, R., Khoshabeh, R., Hollan, J., Borchers, J.: SLAP widgets. In: CHI 2009, p. 481 (2009) 